LUIGI BOBBIO, La democrazia non abita a Gordio. Studio sui processi decisionali politico-amministrativi, Milano, Franco Angeli, 1996, pp. 108.

Si tratta di un piccolo e denso volume nel quale si è concretizzato l'interesse precipuo dell'A. a scavare oltre i processi della rappresentanza degli interessi e del funzionamento del sistema politico, per concentrarsi piuttosto sulle strategie decisionali, sui processi che cercano di trasformare le scelte di governo in mutamento sociale. Bobbio segue qui un «percorso a tre stadi»: esamina e rilancia le obiezioni avanzate «contro l'ipotesi della decisione lineare e del governo razionale»; riflette sulle trasformazioni istituzionali in corso nelle pubbliche amministrazioni, scorgendone il tratto unificante in una crescente frammentazione, territoriale e gestionale, dei poteri pubblici; e avanza, infine, una sua proposta per uno schema di analisi delle decisioni pubbliche, sulla base di uno studio specifico condotto su un numero molto ampio di casi di trasformazione territoriale relativi al Piemonte.

Il libro ci vuole ricordare, in primo luogo che, appunto, la democrazia non abita a Gordio. Si deve sfuggire alla tentazione di adottare modelli di spiegazione troppo forti, che finiscono sempre con il generare spiegazioni troppo povere: intendere la capacità di governare soltanto come l'«assumersi la responsabilità di prendere una decisione» rischia di essere riduttivo e inadeguato in contesti di incertezza, ambiguità e estrema frammentazione come sono quelli in cui vengono solitamente prese le decisioni pubbliche. Contesti nei quali, perciò, il decisore deve piuttosto qualificarsi come un «addetto al montaggio» delle risorse controllate dalla molteplicità degli attori interessati al processo, capace di disegnare il processo stesso, adattandolo o modificando allo scopo di favorire la cooperazione tra gli attori. I nodi che devono essere sciolti nei processi delle decisioni pubbliche sono solitamente formati da intrecci di interessi tali che non possono essere affrontati usando metodi tranchantes, pena il ritrovarsi al punto di partenza o l'aprirsi di problemi maggiori di quelli che si è tentato di risolvere con soluzioni traumatiche.

Bobbio abbozza, invece, un'ipotesi di processo decisionale che assume come dato costitutivo del processo stesso l'intreccio tra gli aspetti tecnici e quelli più propriamente politici, «interattivi». E tende a rimarcare perciò che accanto allo schema previsto dal modello della razionalità «di tipo sostanziale» - nella quale il decisore deve soprattutto essere un «esperto del problema», in possesso di competenze tecniche e capace di stabilire relazioni causa-effetto raccogliendo e organizzando le informazioni pertinenti -, il decisore deve assumere in sé anche competenze «di tipo procedurale», deve essere un «esperto del processo», possessore di competenze interattive. Ed anche queste competenze, che rendono il decisore capace di disegnare il processo e di tenerlo sotto controllo, sono competenze professionali che «possono essere studiate, apprese e trasmesse». 
La figura del decisore così profilata vorrebbe essere antitetica a quella del mediatore politico, «caratteristica della Prima Repubblica italiana». Al mediatore-attore della «consociazione paternalistica», che si sovrappone ai contendenti imponendo loro soluzioni di compromesso che hanno come obiettivo prioritario quello di rafforzare il mediatore stesso nella sua posizione di potere, si contrappone il mediatoreattore della mediazione pluralistica, che assiste le parti e non si sostituisce ad esse, agisce con il loro incarico o con il loro assenso e cerca di facilitarne le interazioni «strutturando il processo di negoziazione».

Insomma, in questa incerta fase di avvio della Seconda Repubblica, si tratta, per Bobbio, di riuscire a trasformare il policy style consensuale ma politicistico della Prima Repubblica in un policy style consensuale ma pluralistico. L'autore ritiene infatti che sia più «realistico» cercare di abbandonare non solo la propensione, tipica del "vecchio" modo di governare in Italia, a ricercare il consenso come tale, quanto le specifiche modalità, «paternalistiche, politicistiche e ristrette con cui la ricerca del consenso è stata praticata». In effetti, la via d'uscita del decisore-autorità, del decisore unico che cerchi di stroncare la tendenza al compromesso, non sembra avere molte possibilità di concretizzarsi, ammesso che sia auspicabile. Meglio puntare su governi che operino con lo stile di decisori-registi, mediatori pluralistici determinati, si può solo auspicare, a liberarsi dei condizionamenti di una tradizione culturale paternalistica «poco propensa a riconoscere l'autonomia dei soggetti sociali».

\section{[Carlo Baccetti]}

ROBERT E. GOODIN (a cura di), The Theory of Institutional Design, New York, Cambridge University Press, 1996, pp. 288.

AREND LIJPHART E CARLOS H. WAISMAN (a cura di), Institutional Design in New Democracies. Eastern Europe and Latin America, Boulder, Colorado, Westview, 1996, pp. 265.

Questi due libri si occupano dello stesso argomento - il disegno delle istituzioni da due prospettive completamente diverse. Il volume collettaneo curato da Robert Goodin affronta la questione dal punto di vista della filosofia e della teoria politica normativa, quello curato da Lijphart e Waisman si colloca, invece, nel mainstream della analisi istituzionale comparata tipico della scienza politica contemporanea. Il problema al centro della raccolta di saggi curata da Lijphart e Waisman è il rapporto esistente tra forma delle istituzioni e processo di transizione verso un regime democratico e una economia aperta di mercato. Quali fattori spiegano la scelta di un dato sistema elettorale, di una data forma di Governo, di un particolare rapporto tra attori 